\title{
Preambolo al Catalogo Sismico Nazionale (CSN) I criteri di informazione del Catalogo Sismico Nazionale (CSN)
}

(Foreward to the National Seismic Catalogue)

(Information criteria of the National Seismic Catalogue)

\author{
R. Console* - C. Gasparini* - B. De Simoni* \\ L. Marcelli* - M. C. SpadeA* - M. VecchI*
}

Ricevuto il 20 Febbraio 1979

ABS'TRACT

This note presents a study meant to set out a national catalogue (C.S.N.) of seismic events the epicentres of which are to be found in Italian territory (or nearby). The catalogue, that will start from the year 1450 B.C., could be used for various purpose employing modern technologies.

The complete C.S.N. is made of three main parts, each of which can also have a separate life:

1) An Analytical Catalogue which comprises the greater part of clata and is the most complete;

2) A Macroseismic Atlas that shows the macroseismic aspect of the most relevant events; Atlas.

3) A Macroseismic Catalogue that translates in numeric terms the

(*) Istituto Nazionale di Geofisica. 
To these is added a comprehensive Bibliography subdivided into 24 chapters each of which covers one of the 24 time periods into which the catalogue has been subdivided.

The Analytical Catalogue besides giving the main parameters for each earthquake (date, epicentre, hypocentral depth, MCS scale intensity, magnitudo, each with its own reliability index) gives indications also on the following sideline data:

1) epicentral location (meaning by this the geographic region and the eventual indication that the epicentre is to be found on a borderline touching more geographic regions, or in the sea, or on the coast, or othermise in the external band (see text);

2) with a reference to the Atlas, the event is shown as having been dealt with also mocroseismic data and it will con be found, as such, in the Atlas and in the Macroseismic Catalogue;

3 ) indications on the typology of the earthquake: i.e. whether it is an "isolated " earthquake, or a "seismic period" (and if so with the indication of the foreshock, main or aftershocks) or of a swarm;

4) the possibility of indicating up to 7 sets of information suitably chose among 40 additional notes regarding instrumental, geophysical, physical or sensorial information besides the possibility of notifying that the event had undergone specific studies or whether it is even to be considered either noth sure or else legendary;

5) the possibility of indicating up to four bibliographical items, is to be considered as capital.

The Macroseismic Atlas gives the isoseists of the more supported by documentary evidences earthquake, with the calculation of the relative areas, of the rays of the equivalent circles and of the rays of prevalent maximal and minimal propagation; these latter with the value of the azimuth measured from the geographical North passing through the macroseismic epicentre.

The Macroseismic Catalogue reports numerically the macroseismic data of the event: analytical and macroseismic epicentre; analytical and macroseismic hypocentral depth, numerical values, in $\mathrm{km}$., of the the three serie of indicated rays, up to isoseist of the 4th degree (MCS).

A fac-simile of working form is given.

\section{RIASSUNTO}

In questa nota viene presentato uno studio inteso ad impostare un catalogo nazionale (C.S.N.) degli eventi sismici i cui epicentri si trovino su territorio italiano (o limitrofo). Il catalogo, che partirà dall'anno 1450 
avanti Cristo, potrà essere utilizzato per vari scopi facendo uso delle tecnologie moderne.

Il C.S.N. completo si articola in tre parti fondamentali, ciascuna delle quali può anche aver vita separata:

1) il Catalogo Analitico che è il più ricco di dati e il più completo;

2) l'Atlante Macrosismico che illustra l'aspetto macrosismico degli eventi più rilevanti; l'Atlante.

3) il Catalogo Macrosismico che traduce in termini numerici

Ad essi si aggiunge una estesa Bibliografia divisa in 24 capitoli ciascuno dei quali copre uno dei 24 archi di tempo in cui è stato suddiviso il catalogo.

Il Catalogo Analitico oltre a dare i parametri fondamentali di ogni terremoto (data, epicentro, profondità ipocentrale, intensità in scala MCS, magnitudo, ciascuno con un proprio indice di attendibilità) dà indicazioni anche sui seguenti dati collaterali:

1) ubicazione epicentrale (intendendo per tale la regione geografica e l'eventuale indicazione che l'epicentro si trova in zona di confine tra più regioni geografiche, oppure epicentro in mare, o sulle coste, o nella fascia esterna) (vedi testo);

2) un rinvio all'Atlante indica che l'evento è stato trattato anche macrosismicamente e lo si ritroverà, sotto questi aspetti, nell'Atlante e nel Catalogo Macrosismico;

3) indicazioni sulla tipologia della scossa: se trattasi cioè di scossa isolata, o di periodo sismico (e in tal caso con l'indicazione di scossa premonitoria o principale o replica) o di sciame;

4) la possibilità di indicare fino a 7 informazioni scelte opportunamente tra 40 note aggiuntive riguardanti informazioni strumentali, o geofisiche, o fisiche o sensorie oltre che la possibilità di segnalare che l'evento è stato oggetto di particolari studi o se addirittura è da ritenersi incerto o leggendario;

5) la possibilità di segnalare fino a quattro voci bibliografiche, tre delle quali da ritenersi le principali.

Nell'Atlante Macrosismico sono disegnate le isosiste dei terremoti più documentati, con il calcolo delle relative aree, dei raggi dei cerchi equivalenti e dei raggi di massima e minima propagazione prevalente; questi ultimi con il valore dell'azimut misurato dal Nord geografico condotto per l'epicentro macrosismico.

Il Catalogo Macrosismico riporta in veste numerica i dati macrosismici dell'evento: epicentro analitico e macrosismico; profondità ipocentrale analitica e macrosismica, valori numerici, in $\mathrm{km}$, delle tre serie di raggi indicati, fino all'isosista di grado IV (MCS).

Viene presentato un fac-simile della scheda di lavoro. 
INTRODUZIONE ALLA COMPILAZIONE DEL C.S.N.

Da qualche anno a questa parte è diventato sempre più pressante, nel campo scientifico e tecnico, il problema di una conoscenza più completa della sismicità di una data regione.

Senza nulla togliere al profondo significato e valore della ricerca scientifica intesa ad approfondire la conoscenza su tutti i problemi geofisici attuali, ci si sta orientando anche su studi che sfruttino quanto accaduto nei tempi passati onde valutare il rischio che l'uomo corre di fronte agli eventi naturali ed eventualmente a prevenirli o a studiare il modo di difendersene.

Molti di questi eventi sono da anni sotto il controllo della scienza; venti, piogge, uragani, cicloni, eruzioni vulcaniche ecc.

Molto più difficile invece uno studio simile sugli eventi sismici, in quanto $i$ parametri che concorrono alla determinazione di un terremoto sono molteplici, complessi, e forse non ancora tutti conosciuti: inoltre anche quelli noti sono di difficile controllo. I vari modelli proposti da molti scienziati per affrontare e risolvere $i$ problemi su basi teoriche di fisica-matematica sono "modelli ", validissimi per una trattazione teorica, ma sovente lontani dalla realtà oggettiva.

Comunque la storia sismica di determinate regioni, affiancata da una conoscenza approfondita delle strutture geologiche e geologico-tettoniche delle regioni stesse, costituisce già di per sé un ottimo materiale di informazione che può essere adeguatamente utilizzato per moltissimi tipi di studi.

La avanzata tecnologia moderna consente oggi studi impensabili solo qualche decennio $f a$, però è ovvio che la macchina aiuta l'uomo tanto piu efficacemente quanto più attenta e scrupolosa è la bontà dei dati che l'uomo fornisce, e quanto maggiore inoltre è il numero delle informazioni ad essi associato.

E' proprio per questo motivo che si sta procedendo, in varie parti del mondo, alla compilazione di "Cataloghi Sismici»opportunamente studiati per una efficiente utilizzazione. 


\section{Cataloghi Sismici in Italia}

La storia sismica italiana è ricca di eventi che hanno una loro documentazione a partire da molti secoli prima della nascita di Cristo: risale al 1450 a.C. la notizia di un terremoto avvertito sui Colli Cimini (Mercalli G., 1883), ma ovviamente le notizie che si riferiscono ad epoche così lontane sono scarse, frammentare e di difficile precisazione.

Non sono mancate, in Italia, pregevolissime raccolte di informazioni sull'argomento, alcune delle quali rese preziose per la esposizione degli eventi a carattere cronologico. Ne citiano solo alcune, tra le piit complete, in ordine di stampa:

1652 - Padre Filippo Da Secinara

"Trattato universale di tutti li terremoti occorsi, e noti nel mondo, con li casi infausti ecc. »-L'Aquila, 1652;

1688 - Vincenzo Magnati

"Notizie Istoriche de' Terremoti succeduti ne secoli trascorsi e nel presente, ecc." - Ed. A. Bulifon, Napoli, 1688;

1691 - Marcello Bonito

"Terra tremante, overo continuatione de' terremoti dalla Creazione del mondo sino al tempo presente"-Ed. Parrino-Mutii, Napoli, 1691;

1738 - Ludovico Antonio Muratori

"Rerum Italicum Scriptores»;

1883 - Giuseppe Mercalli

"I terremoti storici italiani " in "Vulcani e fenomeni vulcanici in Italia»-Milano, 1883;

1901 - Mario Baratta

«I terremoti di Italia»-Ed. Bocca, Torino, 1901;

né si possono ignorare gli altri numerosissimi studiosi che hanno contribuito ad arricchire le conoscenze della storia sismica d'Italia (come il Mongitore, il Morigia, il Moroni, il Gatta, il Per- 
rey, il Serpieri ecc....) per i quali rimandiamo alla bibliografia del C.S.N., ma indubbiamente il piu grande sforzo compiuto in questo campo è da attribuirsi a Mario Baratta, l'autore che nell'opera sopra citata ha fornito materiale preziosissino per una conoscenza generale dell'attività sismica in Italia a partire dall'inizio dell'era volgare fino al 1900.

Questo suo lavoro, condotto con appassionato scrupolo, con perizia scientifica, con critiche costruttive, costituisce ancora oggi una fonte insostituibile di notizie.

In questi ultimi decenni $e$ in tempi recentissimi altri autori si sono cimentati in imprese simili, sia a prosecuzione dell'opera del Baratta, come il Cavasino (1935), sia a sua integrazione nel tempo e nello spazio, come il Mercalli in altre sue opere, sia con studi parziali che forniscono però documentazioni limitate: o solo ad alcuni anni (Caloi 1942, De Panfilis 1959, Malaroda-Raimondi 1957), o solo ad alcune regioni (Val Padana, Carnia, Cansiglio, Italia Centrale, Lazio, Sicilia, Italia Nord-Orientale, ecc...),*

I rilevamenti più o meno sistematici dell'attività sismica italiana - con pubblicazioni di bollettini sismici - accompagnano l'avvento delle strumentazioni apposite che cominciano a comparire dopo la metà del secolo scorso. Solo nel 1938, con la nascita dell'Istituto Nazionale di Geofisica, l'Italia può disporre di un scrvizio sismico nazionale con rilevamento di dati strumentali.

Purtroppo questo servizio dové dibattersi, quasi sul nascere, tra le difficoltà createsi nell'ultimo conflitto mondiale il quale ha ostacolato, per non dire che ha reso impossibile, una raccolta completa di informazioni. $E^{\prime}$ da tener presente tuttavia che le stesse difficoltà ha incontrato tutto il mondo scientifico che ha vissuto tale conflitto, per cui lacune e incertezze sono comuni all'intera scala mondiale.

Quando si è cominciato a sentire il bisogno di riprendere in mano l'argomento, un primo tentativo di dare una impostazione organica a tutto il materiale disponibile e reperibile è stato effet-

(*) Autori vari: Alessio-Biagi; Bottari et al.; Caloi; Caloi-Spadea; De Panfilis-Marcelli; Feliziani-Marcelli; Iaccorino et al.; Marcelli- Pannocchia ecc. 
tuato da F. Giorgetti (dell'O.G.S. di Trieste) e da E. Iaccarino (del CNEN) i quali, con il loro "Italian Earthquake Catalogue from the beginning of the Christian Age up to $1968 »$ (ed. 1971) hanno dato l'avvio al primo catalogo sismico italiano predisposto per una elaborazione automatizzata dei dati resa possibile dallo sviluppo tecnologico del dopoguerra.

A questo hanno fatto seguito altri cataloghi che, con alcune modifiche e maggiore accuratezza, ricalcavano le orme già impostate da Giorgetti e Iaccarino; tra $i$ piu importanti citiamo

il "General Catalogue of Italian Earthquakes" (ed. 1972/CNEN) (di M.T. Carrozzo; G. De Visintini; F. Giorgetti; E. Iaccarino);

il "Catalogo dei terremoti italiani dagli anni 1700 al 1972 " di F. Peronaci (dell'ING) (depositato presso il Min. dei LL.PP.;

il Catalogo a cura dell'Osservatorio di Trieste (non pubblicato);

il Catalogo a cura dell'Istituto di Fisica di Bologna (non pubblicato);

il "Earthquake Catalogue of Calabria and Sicily (dagli anni 1783 a 1973) (di M.T. Carrozzo, M. Cosentino, A. Ferlito, F. Giorgetti, G. Patanè, M. Riuscetti, edito dal CNR 1975)

$e$ infine ancor più recentemente

il Catalogo generale a cura dell'ENEL (dagli anni 1000 al 1975).

Ovviamente ogni catalogazione è intesa a migliorare le edizioni precedenti colmandone eventuali lacune ed arricchendole di ulteriori dati reperiti da indagini sempre piu capillari.

\section{MARCELLI}




\section{IL CATALOGO SISMICO NAZIONALE DELL'I.N.G.}

A questo sforzo generale non poteva mancare il contributo dell'ING, anche perché al suo interno esiste già una ricca raccolta di materiale scientifico catalogato ad opera dei vari ricercatori dell'Istituto che hanno dedicato molta, o parte, della loro attività di ricerca a lavori del genere (come $\mathrm{M}$. De Panfilis, P. Caloi, C. Gasparini, L. Marcelli, G. Pannocchia, F. Peronaci, M.C. Spadea, P.E. Valle, ed altri).

Per quanto il contributo dell'ING sia presente in tutti gli sforzi compiuti dopo il 1960 dai vari operatori citati (avendo l'ING messo a loro disposizione bollettini, note scientifiche e documentazioni (varie) pure ci si sta attualmente impegnando in un'opera di rielaborazione generale finalizzata alla compilazione di un Catalogo Sismico Nazionale (confrontabile o integrabile) con gli altri, che possa offrire garanzie di accuratezza, di completezza e di omogeneità.

A tale scopo il gruppo di studio dell'ING si è riunito varie volte per cercare di definire nei minimi particolari tutti i parametri utili e necessari per rendere le informazioni date utilizzabili sia dal punto di vista scientifico, sia dal punto di vista tecnico.

Esponiamo qui di seguito i criteri seguiti nella scelta dei parametri e delle valutazioni di attendibilità che ad essi si attribuiscono.

Per soddisfare varie esigenze si è riscontrata l'opportunità di articolare il C.S.N. in tre parti fondamentali:

$$
\begin{aligned}
& 1 \text { - CATALOGO ANALitico (C/A) e BIBLIOGRAFIA } \\
& 2 \text { - ATLANTE MACROSISMICO (A/M) } \\
& 3 \text { - CATALOGO MACROSISMICO (C/M) }
\end{aligned}
$$

ciascuna delle quali può avere vita indipendente dalle altre due; però nel loro insieme costituiscono una fonte di informazioni che si è cercato di rendere completa il più possibile. 


\section{Catalogo analitico (C.A.)}

Esponiamo qui di seguito i criteri seguiti nella scelta dei parametri e delle valutazioni di attendibilità che ad essi si riferiscono.

L'elenco sintetico degli elementi forniti è il seguente:

1.1. Data ...... : (Anno, mese, giorno)

1.2. Tempo origine .: (Ore, minuti, secondi) (in tempo Greenwich) (con indice di attendibilità)

1.3. Epicentro ....: ( $\varphi /$ Nord, $\lambda /$ Est) (in gradi e millesimi di grado con indice di attendibilità)

1.4. Profondità ipoc.: $(h) \ldots . . . .$. (in $\mathrm{km}$ ) (con indice di attendibilità)

1.5. Regione geografica (sede dell'evento)

1.5'. Indicazione epicentrale particolare (mare, coste, fascia esterna)

1.5".Località geografica maggiomente colpita (a parole)

1.6. Intensità macrosismica (in scala MCS) (con indice di attendibilità)

1.7. Esistenza di isosiste con riferimento all'Atlante Macrosismico

1.8. Magnitudo $M_{L}, M_{B}, M_{S}$

1.9. Tipologia delle scosse:

9a) Isolata

9b) Periodo sismico

9c) Sciame

1.10. Notizie aggiuntive riguardanti caratteristiche ed effetti connessi al terremoto.

1.11. Bibliografia. 
Osserviamo a questo punto che il C.S.N. si propone di presentare in catalogo tutti i terremoti di cui si sono potute avere notizie, a partire dall'anno 1450 avanti Cristo.

Per molti di essi si ricorrerà alle fonti originali, per molti altri vanno ricercate documentazioni presso varie altre fonti che saranno citate nel punto 1.11. del catalogo (Bibliografia).

E' superfluo dire che molti degli eventi più antichi, avendo a loro sostegno solo le cronache del tempo, risentono, per la loro documentazione, dell'incidenza del periodo storico e politico, dell'evoluzione culturale, degli accentramenti etnici e così via, per cui gli elementi che se ne traggono portano di per sé motivi di incertezza. Tuttavia molti di essi sono documentati con narrazioni così particolareggiate che sopprimere queste informazioni da un catalogo generale sarebbe un voler mutilare la storia sismica dell'Italia.

D'altronde se solo si pensa che tutto ciò di cui è giunta notizia fino a noi ha indubbiamente superato gli errori di valutazione legati agli eventi sismici di entità trascurabile, ci sembra giusto, proprio per una maggiore completezza del catalogo, non ignorare tali informazioni.

L'utilizzatore del catalogo potrà, se riterrà opportuno, operare tutti quei tagli che crederà utili ai fini delle ricerche che si propone di fare.

D'altra parte l'ING ha studiato una serie di programmi (ed altri ancora se ne studieranno) per una molteplice utilizzazione dei dati catalogati.

Gli elementi del Catalogo «C.S.N. » - Analisi critica

\subsection{Data:}

La data completa dell'evento prevede l'anno, il mese, il giorno. Non è previsto alcun indice di attendibilità poiché ove non si conosca il giorno o il mese, al loro posto si mette il simbolo "- con il significato "sconosciuto" $\left({ }^{*}\right)$.

(*) N.B. - Lo stesso significato di "sconosciuto » varrà ogni volta che si incontra il simbolo "-", riferito a qualunque altro elemento. 
Se risultano incerti il giorno o il mese o addirittura l'anno come può verificarsi per alcuni terremoti antichi, si esprimerà tale incertezza con un simbolo apposito:

$\mathrm{H}$ esprime l'incertezza del giorno

I esprime l'incertezza del mese

L esprime l'incertezza dell'anno (vedi scheda di lavoro).

1.2. Tempo origine (in ore Greenwich) e indice di attendibilità

L'indicazione completa del tempo origine prevede:

ora (in tempo Greenwich)

minuti primi

minuti secondi (senza decimi)

indice di attendibilità.

$\mathrm{E}^{\prime}$ ovvio che una tale indicazione potrà essere completa solo per gli eventi rilevati anche strumentalmente, in quanto il tempo origine di un terremoto è uno dei parametri che risultano dall'analisi comparata di varie registrazioni e dal tipo delle dromocrone usate.

L'indice di attendibilità

A, B, C, D, E, F, $\longrightarrow$ per i dati analitici, o $\mathrm{R}, \mathrm{S}, \mathrm{T}, \mathrm{U}, \mathrm{M}, \longrightarrow$ per quelli macrosismici

studiato come segue servirà a discriminare i dati strumentali da quelli macrosismici:

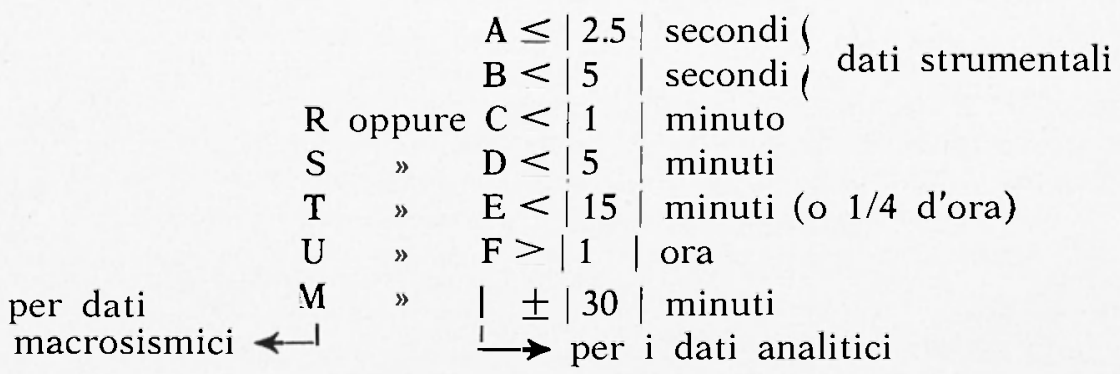


Per quanto riguarda il parametro tempo origine $(\mathrm{H})$ relatitvo agli eventi anteriori al 1894 (data in cui entra in vigore per legge l'adozione in tutta Italia del tempo medio di Roma riferito al meridiano passante per l'Osservatorio del Collegio Romano) valgono le considerazioni desunte da uno studio accurato svolto recentemente sulla "Evoluzione storica delle misure orarie in Italia » (Dominici, Marcelli, 1979), studio al quale rimandiamo per maggiori dettagli: qui ci limitiamo a riportarne le conclusioni con i suggerimenti dati sull'entità degli errori che si ritiene dover attribuire alle misure orarie.

« Da quanto esposto (nella nota citata) risultano con molta chiarezza le difficoltà che si incontrano quando si vogliano tradurre in termini corretti di Tempo Greenwich (TU) le notizie di dati orari di eventi storici (o anche soltanto di quelli anteriori all'avvento dei segnali trasmessi via radio): siano esse quelle pervenute a noi dalle fonti originali, siano pure quelle pervenuteci attraverso interpretazioni di scienziati più o meno attenti al problema.

Comunque, poiché sia nell'un caso che nell'altro ci si trova di fronte a incertezze di base generalmente notevoli, riteniamo utile sintetizzare nei passi seguenti il procedimento di massima da seguire per convertire $i$ tempi degli eventi storici in ore attuali (che sono ore civili di tempo medio) $e$ poi in tempi medi (di Roma o) di Greenwich: diamo altresì un quadro degli errori da attribuire alle misure orarie degli eventi storici (non strumentali) secondo i suggerimenti scaturiti dall'analisi critica condotta (nella nota).

Per le notizie orarie date prima del 1400 è da presumere che fossero adottate le ore temporarie (computate da un tramonto all'altro).

Dopo il 1400 entrano nell'uso quasi generale, e perdurano fin verso il 1860 , le ore italiane (computate da un'avemaria all'altra).

Sia le une che le altre venivano date in tempo vero.

Il primo passo per la conversione dei tempi in ore attuali va fatto con l'ausilio dell'Appendice $D$ (che dà le coordinate geografiche delle città italiane capoluogo di provincia), dell'Appendice $E$ (che dà il tempo civile medio di inizio del giorno alle varie latitudini geografiche nei vari giorni dell'anno e l'equazione del tempor e della figura 7 (che dà una sorta di orologio da usare per una rapida conversione approssimativa: una conversione più accurata si ottiene con $\mathrm{i}$ calcoli numerici indicati dalle formule 20 e 21$\left.){ }^{*}\right)$.

Particolare attenzione va posta, in questa fase, alla eventualità di un cambiamento di data.

(*) Appendici e figura nella nota citata. 
Ricordiamo che solo in tempi più recenti, intorno al 1816, cominciano a comparire (ma non generalmente) misure date in tempi medi locali, per cui va posta molta attenzione all'informazione di base che può essere data sia in tempo vero locale (t.v.l.) sia in tempo medio locale (t.m.l.), sia in tempo medio Roma (t.m. Roma).

Per avere il tempo dell'evento riferito al meridiano di Roma o a quello di Greenwich, fino al 1894 si può operare come segue:

I) se il tempo dell'evento avvertito nel luogo $A(\varphi, \lambda)$ è dato in t.v.l. (ore temporarie, ore italiane, ore a.m. o p.m.) esso va convertito prima in t.m.l., indi in t.m. di Roma, poi in $T U$ mediante il seguente procedimento:

$$
\begin{aligned}
& \text { t.m.l. }=\text { t.v.l. }- \text { Eq. tempo } \\
& \text { t.m. Roma }=t . m . l . \pm \lambda_{\wedge} \text { (in minuti da Roma) }
\end{aligned}
$$

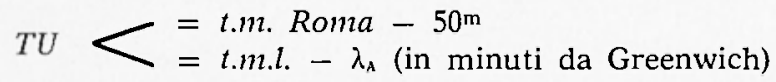

II) se il tempo dell'evento avvertito nel luogo $A$ è dato in tempo medio locale $(t . m . l$.) vale il processo più rapido:

$$
\begin{aligned}
\text { t.m. Roma } & =\text { t.m.l. } \pm \lambda_{\mathrm{A}} \text { (in minuti da Roma) } \\
\text { TII } & =\text { t.m. Roma }-50^{\mathrm{m}} \\
& =t . m . l . ~-\lambda_{\mathrm{A}} \text { (in minuti da Greenwich) }
\end{aligned}
$$

In entrambi i casi va assegnata al $T U$ una attendibilità che viene sintetizzata (in linea di massima) nel modo seguente: in $T U$

se la misura è data in ore temporarie varrà, dopo la conversione

$$
H=T U \pm 45^{\mathrm{m}}
$$

(l'errore si può ridurre se si hanno elementi di valutazione più validi);

in tutti gli altri casi (ore italiane, ore a.m. o p.m.) varrà):

$$
\begin{aligned}
& \underset{\text { del }}{\operatorname{prima}} H-T U+30 \mathrm{~m} \text { (se la misura originale è data in ore intere) } \\
& \text { del } 1866=T U \pm 15 \mathrm{~m} \quad \text { (se la misura originale è data al quarto d'ora) }
\end{aligned}
$$

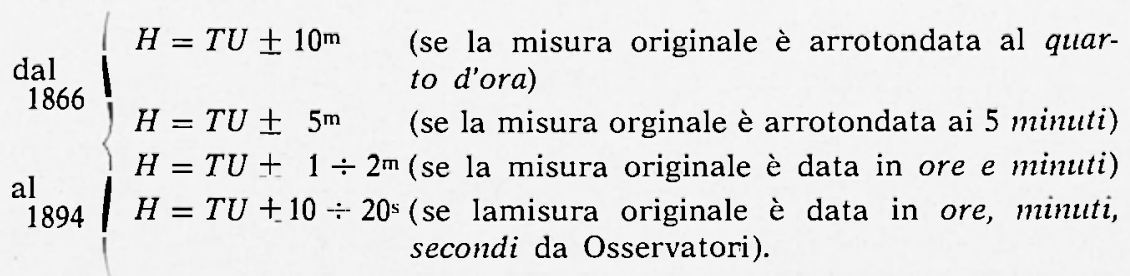

(*) Vale il segno + se $A$ è ad Ovest di Roma, il segno - se $A$ è ad Est di Roma. 
Per gli eventi posteriori al 1894 per lo piì si tratta di dati strumentali forniti da Osservatori sismologici, sicché valgono le norme illustrate (all'inizio del presente paragrafo).

Va sempre ricordato che i tempi che inizialmente sonoriferiti al $t . m$. di Roma diventano tempi Greenwich $(T U)$ togliendo dal tempo di Roma 50 minuti:

$$
49,8^{m} \approx 50^{m}=\lambda \text { Roma (da Greenwich) }
$$

\subsection{Epicentro $(\varphi, \lambda)$ e indice di attendibilità}

L'indicazione completa dell'epicentro prevede

$\varphi=$ latitudine geografica Nord espressa in gradi e millesimi di grado $\left(0,000^{\circ}\right)$

$\lambda=$ longitudine Est $\mathrm{di}$ Greenwich espressa in gradi $\mathrm{e}$ millesimi di grado $\left(0,000^{\circ}\right)$

Indice di attendibilità.

Anche qui è da notare che la terza cifra decimale di $\varphi$ e di $\lambda$ acquista significato solo per gli epicentri calcolati strumentalmente o rilevati da studi appositi.

Comunque anche in questo caso la precisione dell'informazione viene messa in evidenza dall'indice di attendibilità il quale sarà dato da un raggio " $r$ » in $\mathrm{km}$ intorno all'epicentro $(\varphi, \lambda)$ valutato come segue (Marcelli, Pannocchia, 1974) per valutazioni dell'epicentro rispettivamente macrosismiche o analitiche.




E' ovvio che per le date anteriori al 1872 (data in cui cominciano a comparire i primi rilevamenti strumentali più o meno sistematici) l'informazione epicentrale sarà esclusivamente macrosismica e verrà assunto come epicentro il baricentro della isosista di intensità più elevata (quando c'è la possibilità di una tale delineazione) oppure le coordinate della località che ha segnalato l'evento quando manchino altre indicazioni (per esempio... « terremoto forte [. $\equiv \mathrm{V}$ MCS] sentito a Siena).

Dopo il 1872 le due indagini parallele (analitica e macrosismica) potrebbero condurre a risultati diversi per la ubicazione epicentrale. In tali circostanze verranno riportati entrambi gli epicentri, ciascuno con il proprio indice di attendibilità.

Ricordiamo a questo punto che poiché il catalogo ING prevede anche la elaborazione di un Atlante Macrosismico di cui parleremo tra breve, ivi verranno riportate, insieme con molte informazioni che riguardano l'aspetto macrosismico dell'evento, anche la posizione dei due epicentri e poiché la maggior parte di queste informazioni sarà catalogata nel Catalogo Macrosismi$c o$, anche in questo ultimo compariranno, quando sarà necessario, entrambi gli epicentri (vedi punto 3 ).

\subsection{Profondità ipocentrale «h»(in $\mathrm{km})$ e indice di attendibilità}

Questo parametro, pur essendo importantissimo, è uno degli elementi più discutibili in fatto di precisione.

Su questo argomento è stato condotto presso L'ING (Marcelli, Peronaci, 1979) uno studio critico particolareggiato che mette in luce le difficoltà che si incontrano sempre nelle valutazioni di $h$. Un altro studio parallelo è stato condotto per la ricerca di una determinazione rapida di $h$ con metodi macrosismici, utilizzando per il coefficiente " $\gamma$ » (che compare nella formula di Blake) i valori desunti da un'analisi condotta su tutti i terremoti italiani di cui si era desunta la $\alpha h »$ in studi analitici particolari, (Marcelli, Spadea in press).

Qualunque sia il metodo impiegato per il calcolo della profondità ipocentrale (sia analitico, sia macrosismico), si ritiene 
indispensabile valutarne anche l'errore, il quale acquisterà maggior significato se considerato percentualmente.

In base a queste considerazioni, alle " $h$ " riportate in catalogo saranno associati degli indici di attendibilità che esprimeranno il metodo di calcolo e l'errore percentuale, con la seguente simbologia:

$\downarrow$ per « $h$ » calcolato con metodo $-\downarrow$

analitico
A se oh $10 \% h$
B se oh $20 \% h$
C se oh $50 \% h$

macrosismico

D se $0 h 10 \% h$

E se oh $20 \% h$

F se $\delta h 50 \% h$

se $\bar{o} h>50 \% h$ varranno le seguenti indicazioni per « $\mathrm{h}$ » calcolato con l'uno o con l'altro metodo

$\mathrm{X}$ vale per ipocentro sopra la "Conrad» $\left(^{*}\right)$

$\mathrm{Y}$ vale per ipocentro sopra la «Moho»

$\mathrm{Z}$ vale per ipocentro nel « Mantello».

Le valutazioni di $\mathrm{X}, \mathrm{Y}, \mathrm{Z}$ vanno stimate in base a ragionamenti fatti sulle cause dell'errore, oppure in base alle conoscenze sulla natura delle "strutture sismo-tettoniche» (vedi appresso).

Esempi numerici basati sul criterio assunto

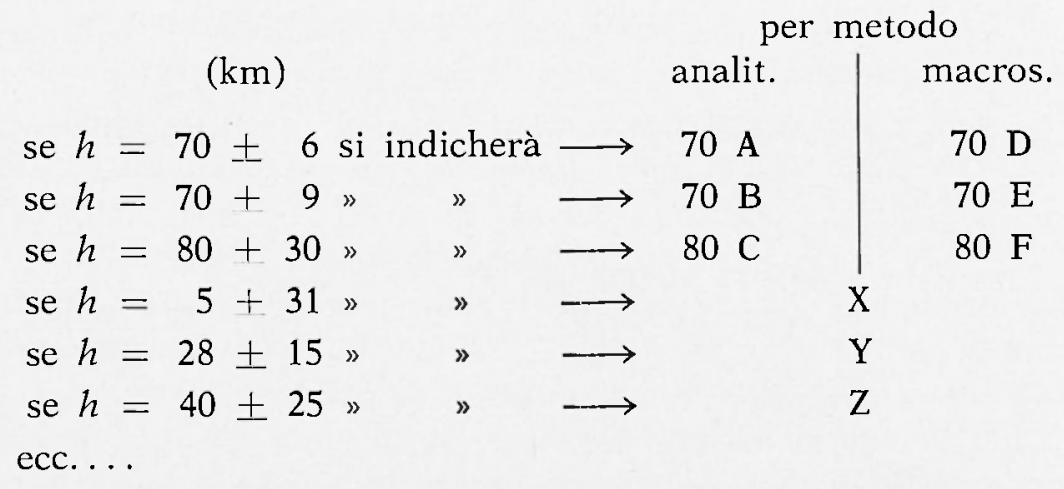

${ }^{*}$ ) Questa indicazione avrà senso per i terremoti studiati in modo particolare. 


\subsection{Unità tettonico-stratigrafiche e regioni geografiche}

Sarebbe nelle nostre intenzioni poter affiancare ad ogni evento anche l'indicazione dell'unità tettonico-stratigrafica in cui esso è avvenuto ritenendo che l'aspetto macrosismico degli eventi sia influenzato dalle varie strutture geologiche, ma non essendo ancora disponibili i risultati delle ricerche sulle carte sismo-tettoniche, tale indicazione si deve per il momento tralasciare, pur prevedendo la possibilità di aggiungere l'informazione quando fosse disponibile.

In vista di possibili utilizzazioni del catalogo anche nel campo politico-amministrativo (come potrebbe essere ad esempio il controllo dell'edilizia a livello regionale) abbiamo previsto una indicazione anche per la regione geografica sede dell'evento. Ogni regione sarà individuata mediante una lettera di codice come nella tabella a pagina seguente.

N.B. Abbiamo indicato con « $\mathrm{K}$ » la zona del basso Tirreno, che non compare nella suddivisione delle regioni, ma che è sede di molti eventi sismici a grande profondità.

\begin{tabular}{|c|c|c|c|}
\hline c & LOMBARDIA & $\mathrm{Q}$ & ABRUZZO \\
\hline D & TRENTINO ALTO ADIGE & $\mathrm{R}$ & MOLISE \\
\hline $\mathrm{E}$ & VENETO & $\mathrm{s}$ & CAMPANIA \\
\hline $\mathrm{F}$ & FRIULI VENEZIA GIULIA & $\mathrm{T}$ & PUGLIA \\
\hline G & LIGURIA & $\mathrm{U}$ & BASILICATA \\
\hline $\mathrm{H}$ & EMILIA ROMAGNA & $\mathrm{V}$ & CALABRIA \\
\hline $\mathrm{L}$ & TOSCANA & W & SICILIA \\
\hline M & MARCHE & $\mathrm{Z}$ & SARDEGNA \\
\hline & BASSO $\mathrm{T}$ & & \\
\hline
\end{tabular}




\subsubsection{Indicazioni epicentrali particolari}

In aggiunta a quanto esposto nel punto 1.5. si è ritenuto utile dare una informazione aggiuntiva riguardante gli eventi che si trovano in mare, o sulle coste o fuori del confine geografico dell'Italia: tale informazione viene indicata con

$M$ quando l'epicentro è sicuramente in mare

$\mathrm{P}$ quando l'epicentro è probabilmente in mare o sulle coste

E quando l'epicentro è nella fascia esterna che allarga la zona esplorata, come indicato nella figura 1.

$\mathrm{R}$ quando l'epicentro è in zona di confine tra due o più regioni geografiche.

A proposito dell'indicazione « $E$ » va notato che saranno riportati in catalogo solo quei terremoti che, pur con epicentro fuori del territorio nazionale hanno avuto qualche ripercussione macrosismica su località italiane.

(Per esempio il terremoto di Cefalonia del 12 agosto 1953 è stato avvertito macrosismicamente - di III-IV - sulle coste adriatiche, a sud del golfo di Manfredonia).

N.B. In tal caso la intensità riportata sarà la massima avvertita sulle località italiane, come verrà precisato al punto 1.6.

\subsubsection{Località geografica (a parole)}

Per dare al catalogo anche la possibilità di una facile consultazione, viene indicata a parole, la località epicentrale geografica maggiormente interessata dall'evento.

(Per esempio Verzegnis-Tolmezzo - Friuli).

1.6. Intensità macrosismica (in scala M.C.S.) e indici di attendibilità

L'intensità macrosismica, in gradi M.C.S., viene indicata con il numero corrispondente al grado massimo.

Se il terremoto è stato soltanto registrato (ma non avvertito) in prossimità dell'epicentro allora è posto $I=1$ (come ad 




Figura 1 - Carta d'Italia - Codici delle regioni e limiti della fascia esterna.

esempio nel caso di piccole scosse dei periodi sismici del Friuli o di Ancona).

Poiché è indispensabile individuare dal catalogo se la intensità riportata sia riferita o meno all'epicentro, si è convenuto di indicare con

"IA » la intensità massima data da informazioni macrosismiche nella zona epicentraale; e con

"IB » la intensità massima avvertita su località italiane nel caso in cui l'epicentro si trovi nella fascia esterna, oppure in mare, o che comunque non cia riferita all'epicentro. 
Indici di attendibilità sono associati a ciascun valore della $I A$ e della $I \mathrm{~B}$, con i seguenti simboli e significati:

- vale per attendibilità massima, relativa ad un'area megasismica con $r \leq 6 \mathrm{Km}$ (conformemente ai primi due gradi di attendibilità dell'epicentro sopra descritti:

$$
\begin{aligned}
& \mathrm{P}|\mathrm{O}| r \leq 2 \mathrm{Km} \\
& \mathrm{Q}|\mathrm{A}| r<6 \mathrm{Km}
\end{aligned}
$$

$\mathrm{X}$ vale per $+1 / 2$ grado della M.C.S.

$\mathrm{Y}$ vale per \pm 1 grado della M.C.S.

$\mathrm{W}$ vale per $>$ della $I$ indicata

Rimarrà "- " (sconosciuta) quando non sia possibile valutarne il grado sia per mancanza di notizie, sia per notizie incerte o contrastanti.

Esempi:

1) $10.5 \mathrm{AX}$ si interpreta come l'intensità massima di un terremoto a terra (A) cui sia stata riconosciuta una $I_{0}=10-11(=10.5)$ con attendibilità $\mathrm{X}$, pari cioè a $1 / 2$ grado: ossia $I_{0}=10.5 \pm 0.5$ (M.C.S.).

2) $5.5 \mathrm{BY}$ si interpreta come segue: l'epicentro del terremoto si trova in mare, oppure nella fascia esterna, ma è stato avvertito su località del territorio italiano (e quindi in zona non epicentrale " B ") con una intensità massima $5 \frac{1}{2}$ (M.C.S.) e con una incertezza di +1 grado $(\mathrm{Y})$, talché potrebbe essere 4.5 oppure 6.5.

3) $7.5 \mathrm{AW}$ si interpreta come segue: l'epicentro è su territorio nazionale $(\mathrm{A})$ : l'intensità segnalata è $7 \frac{1}{2}$, ma all'epicentro potrebbe essere maggiore.

Osservazioni - L'indicazione delle intensità è sempre data in base a valutazioni dirette $\left(^{*}\right)$. Si è preferito tralasciare l'in-

$\left.{ }^{\star}\right)$ desunte dalla descrizione macrosismica dell'evento o da altri elementi di valutazione (per esempio i terremoti che non compaiono nelle descrizioni del Baratta sono da ritenersi per lo più d'intensità inferiore al VI grado). 
mule in uso che legano tra loro vari parametri sismici, quali la magnitudo, l'intensità, l'energia, la profondità ipocentrale. formazione piuttosto che ricorrere a qualcuna delle tante forCon questo si è voluto evitare il rischio di introdurre degli errori arbitrari che possono essere contenuti in relazione teoricosperimentali suscettibili di perfezionamento.

Saranno studiati dei programmi di elaborazione dei dati originali introdotti in catalogo, e ciò consentirà di utilizzare $i$ dati stessi in parallelo con il progresso della ricerca scientifica.

\subsection{Isosiste}

Tutti i terremoti per i quali è stato possibile studiare l'aspetto macrosismico, hanno una indicazione che fa riferimento all'esistenza delle isosiste (vedi punto 2: "Atlante macrosismico»).

\subsection{Magnitudo}

Per ogni terremoto sono previsti tre valori della magnitudo:

$M L$ (indica il valore della magnitudo calcolata strumentalmente entro $600 \mathrm{~km}$ di distanza dall'epicentro, mediante sismografi Wood Anderson, o Wiechert, o strumenti antichi sull'uso dei quali, a questo scopo, è stata pubblicata recentemente una nota (Casal, Console, De Simoni, 1977). I valori $M L$ saranno tutti tratti dai calcoli diretti sulle registrazioni.

$M B$ (indica il valore della magnitudo riportata dal bollettino internazionale dell'I.S.C.).

MS (indica il valore della magnitudo tratto da pubblicazioni o da altri cataloghi o da metodi macrosismici [secondo la relazione $M_{F}=f\left(I_{\mathrm{o}}, h\right)$ ] (Basili, Marcelli, Smriglio, in press).

Ciascuna delle tre magnitudo va mediata, se possibile, tra più valori corrispondenti. 
Non è previsto alcun indice di attendibilità; i valori saranno limitati ad una sola cifra decimale, approssimata per difetto. Se i cataloghi originali riportano valori a frazione $n / 4$ come per esempio $5 \frac{1}{4}, 6 \frac{3 / 4}{2} \ldots$ varranno rispettivamente $5.2 ; 6.7 \ldots$

Osservazioni - Anche qui vale quanto detto a proposito delle intensità.

$E^{\prime}$ da prevedere che la colonna delle magnitudo (specie quella delle $M_{L}$ ) sarà documentata solo per i terremoti di questo ultimo secolo e per qualcuno datato fine 1800. Anche se ciò comporterà un grande vuoto di dati di magnitudo, si è preferito attenersi ai soli dati originali piuttosto che introdurre in veste definitiva valori di $M$ che potrebbero in futuro risultare inesatti: eventuali conversioni potranno essere programmate all'interno del calcolatore.

\subsection{Tipologia delle scosse}

Ci è sembrato importante introdurre nel catalogo alcune indicazioni riguardanti la tipologia degli eventi sismici: questa potrebbe rappresentare una caratteristica sismica del territorio interessato, utile alla conoscenza dell'abito sismico di determinate zone.

Gli eventi sismici possono presentarsi in tre forme diverse:

9a) come scosse isolate (isol.)

9b) come periodi sismici (F, P, A)

9c) come sciami

\subsubsection{Scosse isolate}

Gli eventi del tipo 9a) "scosse isolate", sono piuttosto rari; essi possono tuttavia verificarsi: citiamo come esempio il terremoto di Tuscania del giugno 1974. Se poi si prescinde da una eventuale microsismicità non percettibile all'uomo, la quale potrebbe essere messa in evidenza solo da strumentazioni sensibili appositamente installate intorno alla zona epicentrale, ne 
segue che la documentazione relativa al numero degli eventi di tipo 9a) risulterà molto più elevata di quanto non sia in realtà.

Queste considerazioni valgono in modo particolare per i terremoti storici, privi di qualsiasi documentazione strumentale.

Si è convenuto che nel catalogo C.S.N. siano indicate come "scosse isolate» contrassegnate con "isol» quelle che, o per mancanza di notizie o per qualche altro motivo, non si possano associare ad un periodo sismico.

Per quanto esposto va da sé che nei primi secoli abbonderanno le indicazioni di scosse isolate. Talché "isol " vale per scossa isolata o notizia isolata.

\subsubsection{Periodi sismici}

Gli eventi del tipo $9 b$ ) "periodi sismici» sono invece di gran lunga i più numerosi.

Ricordiamo che i periodi sismici sono caratterizzati dal verificarsi di una o più scosse principali e da una serie di scosse minori; la prima scossa principale può essere preceduta o meno da alcune scosse cosiddette "premonitorie".

Si sa che non è facile definire rigorosamente i limiti di tempo e di spazio di un periodo sismico. Per il C.S.N. se ne è data la seguente definizione che vale come una convenzione assunta per il catologo (ed a cui ci si attiene quando mancano indicazioni esplicite).

Per «periodo sismico" si intende un insieme di $n$ scosse provenienti dalla stessa origine, vincolate dai seguenti legami di tempo e di spazio.

Legami di tempo:

a) per la durata delle repliche si fa riferimento generico ai valori della tabella 1 dedotta da Gardner e Knopoff (1974) in uno studio condotto su alcuni periodi sismici californiani.

b) la ricerca delle scosse premonitorie va estesa fino ad intervallo di tempo $\tau_{F}$ (prima della prima scossa principale) pari ad 1/10 della durata delle repliche, secondo un criterio ipotizzato a suo tempo da Cancani (1902) (vedi figura 2). 


\section{TABELLA 1}

Periodi sismici: durata delle repliche in funzione della magnitudo della scossa principale.

\begin{tabular}{|c|c|c|c|c|}
\hline$M$ & $\begin{array}{c}\tau_{\text {A (in giorni) }} \\
\text { (durata delle } \\
\text { repliche) }\end{array}$ & & $M$ & $\begin{array}{c}\tau_{\text {A }} \text { (in giorni) } \\
\text { (durata delle } \\
\text { repliche) }\end{array}$ \\
\hline 2.5 & 6 & 5.5 & 290 \\
3.0 & 11.5 & 6.0 & 510 \\
3.5 & 22 & 6.5 & 790 \\
4.0 & 42 & 7.0 & 915 \\
4.5 & 83 & 7.5 & 960 \\
5.0 & 155 & 8.0 & 985 \\
\hline
\end{tabular}

Legami di spazio:

c) le repliche che vanno ricercate entro un'area " $A$ " intorno all'epicentro della scossa principale che ovviamente è funzione delle magnitudo.

Analogamente a quanto proposto da alcuni studiosi (Utsu, Seki, Tsuboi, Bath, Duda e altri ancor più recentemente) varrà anche per noi una relazione del tipo

$$
\log A=a M+b
$$

dove ad « $a$ » e a « $b$ » vanno attribuiti opportuni valori numerici. Per il C.S.N. abbiamo scritto la formula di cui sopra ponendo $a=0.82$ e $b=-2$ per cui si ha:

$$
\log A=0.82 M-2
$$

i valori di « $a » \mathrm{e} « b$ sono stati desuti dall'andamento di alcuni tra i più recenti periodi sismici (Ancona, Sicilia Occidentale, Friuli). La [1] è solo un'ipotesi di lavoro che viene assunta per stabilire un primo criterio di selezione.

Nella tabella 2 sono riportati i valori delle aree $A$ (in $\mathrm{kmq}$ ) relative alle varie Magnitudo e due serie di raggi $r_{c}, b_{c}$ corrispon- 


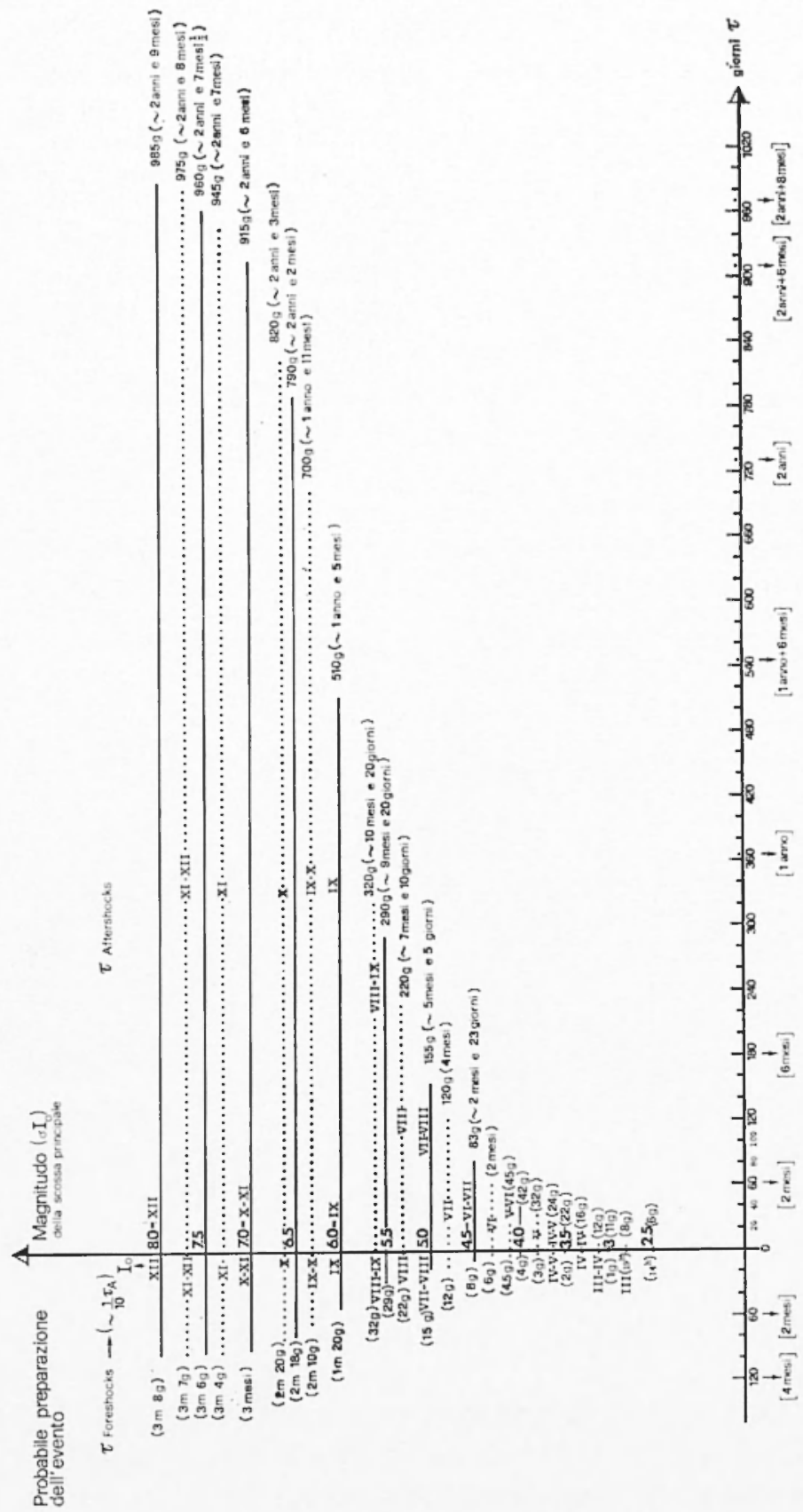

Figura 2 - Durata probabile delle repliche in funzione della magnitudo 
denti: la prima serie " $r_{e}$ » si riferisce all'ipotesi di un'area circolare: la secondo serie $" b_{e}$ » si riferisce all'ipotesi di una equivalente area ellittica con il semiasse maggiore $b_{e}=3 / 2 r_{c}(\mathrm{e}$ il semiasse minore $=2 / 3 r_{c}$ ).

Il criterio di selezione sarà pertanto quello di ricercare le repliche ad una distanza - dall'epicentro della scossa principale - compresa tra $r_{c}$ e $b_{e}$ (vedi grafici di figura 3).

Ciò premesso, esponiamo il criterio seguito nel C.S.N. per evidenziare i concetti esposti.

L'intero periodo di tempo esaminato (dal 1450 avanti Cristo al 1980) viene diviso in 24 intervalli di tempo (1, A, B, C...) (vedi capitolo 1.11 - Bibliografia): all'interno di ciascuno di questi intervalli ogni periodo sismico va indicato con un numero progressivo che si mantiene costante per tutte le scosse appartenenti ad esso; e all'interno di ogni periodo vanno indicate

e cosi via.

con $\mathrm{F}$ tutte le scosse premonitorie

" $\mathrm{P}_{\mathbf{1}} \quad$ la prima scossa principale

" $A_{1}$ la serie delle replice dopo la

" $A_{1}\{$ prima scossa principale

" $\mathrm{P}_{2} \quad$ la seconda scossa principale

" $\mathrm{A}_{2}$ | la serie delle repliche dopo la

» $A_{2}\{$ seconda scossa principale

Conveniamo di considerare $\mathrm{P}_{\mathrm{i}}$ tutte le scosse la cui intensità non differisca più di $1 / 2$ grado M.C.S. dalla $I_{o}$ massima (o non più di 2 decimi di magnitudo).

Esempio: se nel C.S.N. si trovano le indicazioni

\begin{tabular}{|c|c|}
\hline $54 \mathrm{~T} \quad \mathrm{~F}_{1}$ & $54 \mathrm{~T} \mathrm{P}_{2}$ \\
\hline $54 \mathrm{~T} \mathrm{~F}$ & $54 \mathrm{~T} \mathrm{~A}$ ? \\
\hline $54 \mathrm{~T} \quad \mathrm{P}_{1}$ & $54 \mathrm{~T} \mathrm{~A}$ \\
\hline $54 \mathrm{~T} \quad \mathrm{~A}_{\mathrm{I}}$ & $54 \mathrm{~T}$ \\
\hline $54 \mathrm{~T} \quad \mathrm{~A}_{1}$ & \\
\hline
\end{tabular}

esse si interpretano nel modo seguente: si tratta del $54^{\circ}$ periodo relativo all'intervallo di tempo $\mathrm{T}$ (dal 1931 al 1940) del catalogo, in cui compaiono due scosse premonitorie $(F)$; una prima scossa 
principale $P_{1}$ seguita da due repliche $\left(A_{1}\right)$, una seconda scossa principale $P_{2}$, seguita da $n$ repliche $\left(A_{2}\right)$ ecc.
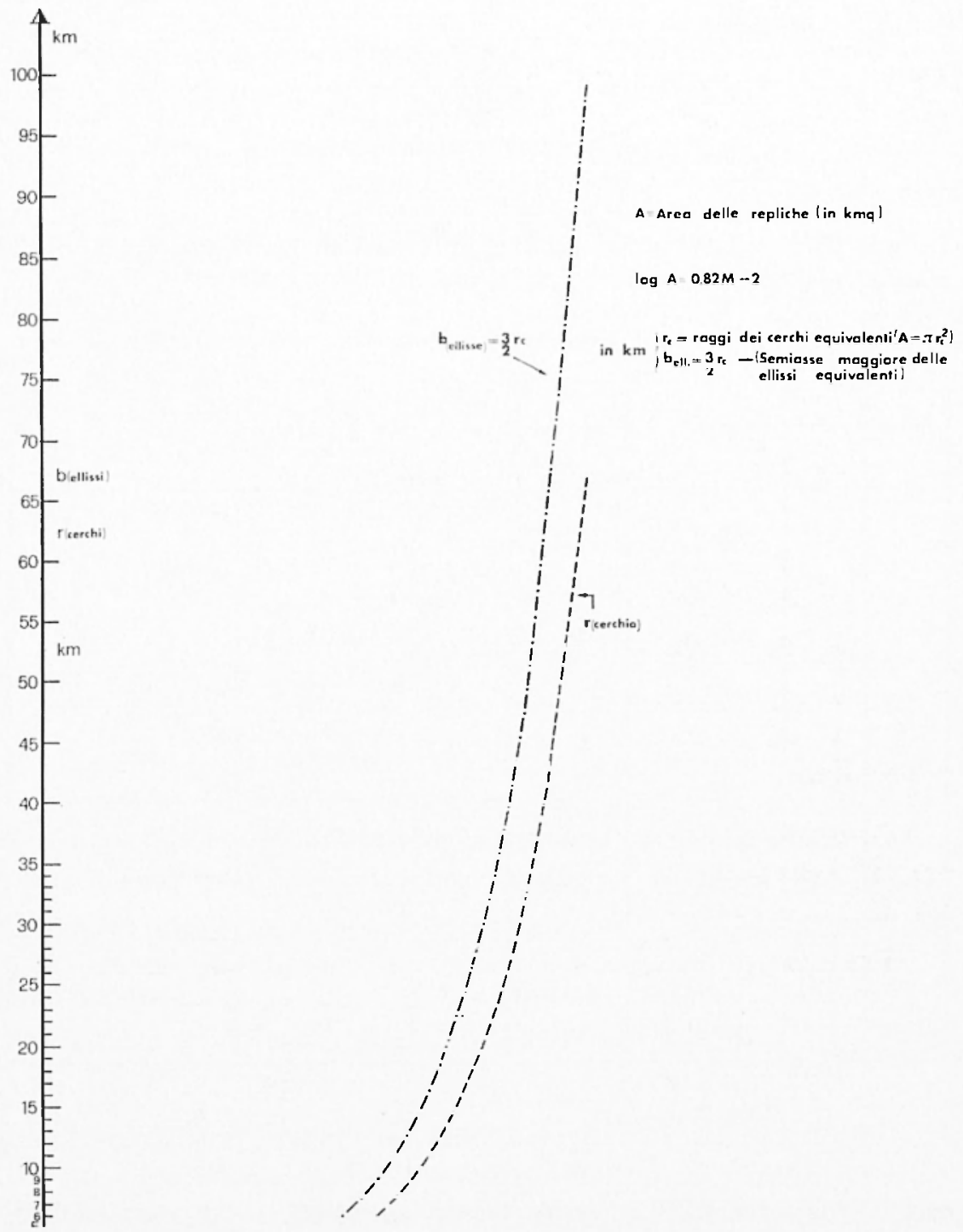

Figura 3 - Area delle repliche in funzione della magnitudo ( $\log A=0.82 M-2$ ). 
E' superfluo notare che nel catalogo generale la continuità del periodo può essere spezzata dall'inserimento di altri eventi sismici cronologicamente interposti, ma la presenza del numero (54 $\mathrm{T}$ nell'esempio) consente una immediata ricostituzione dell'intero periodo.

TABELLA 2

$$
\log A=0.82 M-2
$$

Periodi sismici: area delle repliche (in funzione della magnitudo della scossa principale e serie de raggi corrispondenti ad aree circolari o ellittiche).

\begin{tabular}{|r|r|r|r|rl|l|}
\hline$M$ & $\log A$ & \multicolumn{1}{|c|}{$\begin{array}{c}A \\
(\mathrm{kmq})\end{array}$} & $\begin{array}{c}r_{c} \\
(\mathrm{~km})\end{array}$ & \multicolumn{2}{|c|}{$b_{e}=\frac{1}{2} r_{c}$} & \\
\hline 3.0 & 0.46 & 2.88 & 0.96 & 1.44 & \\
3.5 & 0.87 & 7.41 & 1.54 & 2.30 & \\
4.0 & 1.28 & 19.05 & 2.46 & 3.69 & \\
4.5 & 1.69 & 48.98 & 3.95 & 5.92 & \\
5.0 & 2.10 & 125.89 & 6.33 & 9.50 & Ancona \\
5.5 & 2.51 & 323.59 & 10.15 & 15.22 & Sic. Occid. \\
6.0 & 2.92 & 831.76 & 16.27 & 24.41 & Friuli \\
6.2 & 3.08 & 1213.39 & 19.65 & 29.48 & \\
6.5 & 3.33 & 2137.96 & 26.09 & 39.13 & \\
7.0 & 3.74 & 5495.40 & 41.82 & 62.74 & \\
7.2 & 3.90 & 7943.27 & 50.28 & 75.43 & \\
7.5 & 4.15 & 14125.35 & 67.05 & 100.58 & \\
\hline
\end{tabular}

E' da prevedere l'impossibilità di riportare singolarmente tutte le repliche di un dato periodo sismico: anche nelle descrizioni più dettagliate sovente viene riportato un numero cumulativo e approssimativo delle repliche minori, o addirittura una informazione generica. 
Per non tralasciare questa informazione, che può avere la sua importanza, si è stabilito di riservare a questo scopo 13 delle 40 note aggiuntive previste (vedi punto 1.10.).

I vari raggruppamenti numerici o le varie indicazioni quantitative sono riportate nello schema seguente:

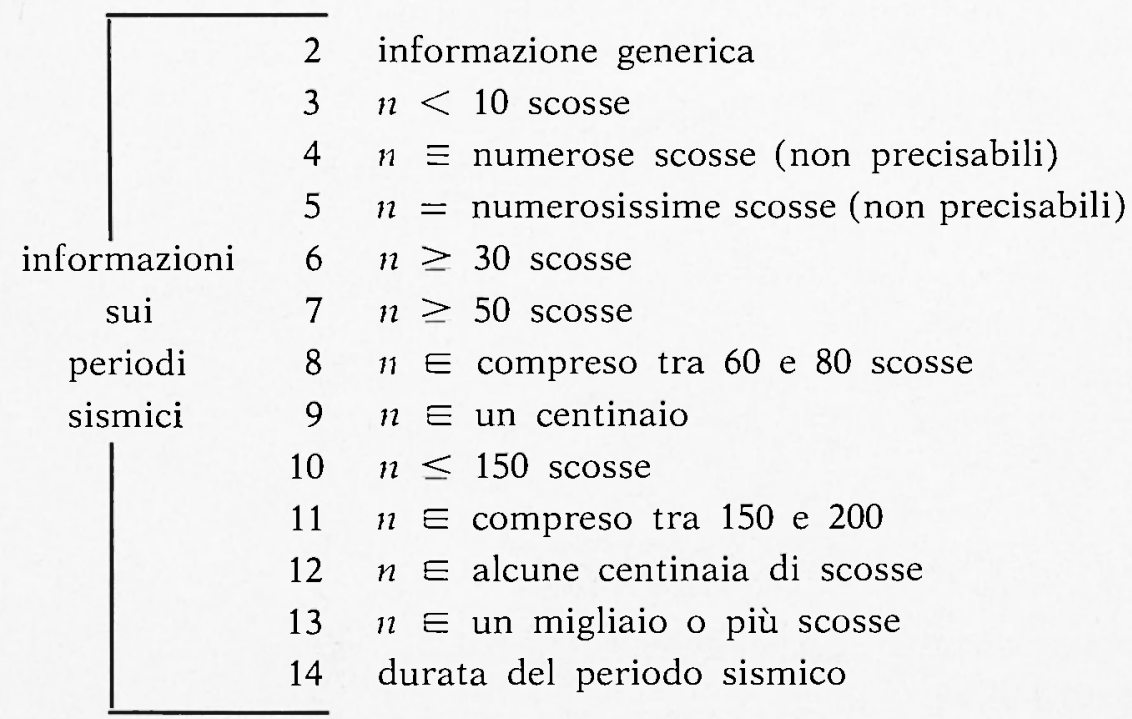

La nota numero 2 dirà che si ha una informazione molto generica del periodo sismico senza possibilità di precisazione (per esempio "si avvertirono repliche per molti giorni »).

Come si vede è prevista anche la possibilità di far sapere se si conosce la durata del periodo sismico (nota 14) ma tale informazione rimarrà archiviata, non essendo possibile trasferirla in scheda.

Il numero delle repliche che compariranno in catalogo sarà per ovvii motivi molto inferiore al numero reale.

\subsubsection{Sciami}

Nel catalogo C.S.N. saranno evidenziati anche gli eventi del tipo 9c) "sciami" cui abbiamo dato la seguente definizione: una sequenza di numerosi eventi di intensità paragonabili tra 
loro e, ovviamente, provenienti da una medesima zona (in genere di intensità moderata).

La loro indicazione nel catalogo va fatta con lo stesso criterio dei periodi sismici: solo che nel caso degli sciami non ci saranno differenziazioni tra le varie scosse.

Ogni sciame sarà indicato con un numero progressivo, con la progressività dei periodi, ma con l'indicazione S.

Esempio: se nel catalogo si legge

$$
\begin{array}{r}
23 \mathrm{Q} \mathrm{F} \\
23 \mathrm{Q} \mathrm{F} \\
23 \mathrm{Q} \mathrm{P} \\
\rightarrow 24 \mathrm{Q} \mathrm{S} \\
\rightarrow 24 \mathrm{Q} \mathrm{S} \\
23 \mathrm{Q} \mathrm{A} \\
\rightarrow 24 \mathrm{Q} \mathrm{S} \\
23 \mathrm{Q} \mathrm{A}
\end{array}
$$

va interpretato dicendo che durante il $23^{\circ}$ periodo sismico dell'intervallo di tempo $Q$ ha avuto inizio uno sciame (il $24^{\circ}$ nella numerazione complessiva periodi + sciami).

\subsection{Note aggiuntive}

Notizie riguardanti caratteristiche ed effetti connessi al terremoto

E' previsto anche un certo numero di notizie che aggiungono ulteriori informazioni a quelle fin qui esposte: serviranno a dare una idea dei fenomeni connessi al terremoto.

Se ne possono segnalare fino a 7 scelte tra le 40 elencate nello schema riportato nella pagina seguente.

La nota 1) dirà che il terremoto è stato studiato in modo particolare, e in tal caso i riferimenti bibliografici (vedi al punto 1.11 ) daranno le indicazioni relative. 
Le note da 2) a 14) sono riservate alle notizie relative ai periodi sismici secondo quanto detto precedentemente.

Le note da 15) a 21) danno informazioni strumentali (sismiche, meteorologiche, magnetiche).

Le note da 22) a 26) danno informazioni sui terreni.

Le note da 27) a 32) danno informazioni sulle acque.

La nota 33) dà informazioni su effetti vari non previsti nelle altre voci.

Le note da 34) a 36) danno informazioni su effetti sensori e fisici.

Le note da 37) a 39) danno informazioni sulle caratteristiche principali degli eventi.

La nota 40) ha il significato di "leggendario o incerto" (questa indicazione si troverà solo in corrispondenza di alcuni eventi storici per i quali non è stato possibile appurare l'esattezza della fonte, come si è detto nella parte introduttiva).

SCIIEMA DELLE NOTE AGgIUNTIVE

1 studiato in modo particolare

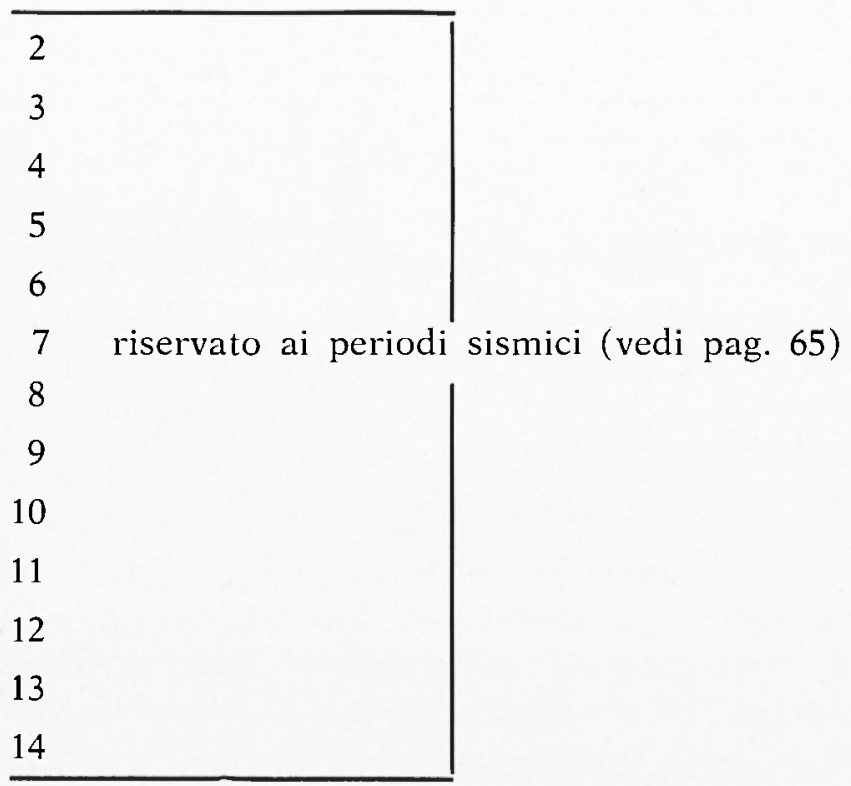


15 esistenza di dati di accelerazione

16 forti anomalie generali nei tempi d'arrivo delle onde $P$; (O-C)

17 non registrato

18 registrato in pochi osservatori (meno di 10)

19 registrato in numerosi osservatori (più di 10)

20 variazioni (strumentali) di pressione atmosferica

21 variazioni (strumentali) di campo magnetico

22 franamento di masse di materiale terroso

23 franamento di masse di materiale roccioso

24 fessurazioni in terreni incoerenti

25 fessurazioni in terreni compatti

26 apertura di voragini

27 espulsioni di sabbie, acque, fango, gas (fontane sismiche)

28 intorbidamento di acque

29 riscaldamento di acque

30 variazioni di portata o livello dell'acqua in fiumi, laghi, pozzi...

31 nascita di nuove sorgenti o disseccamento di vecchie

32 nascita di sorgenti termiche

33 effetti vari

34 inquietudine negli animali e/o variazioni di temperatura ambiente

35 rombi

36 lampi sismici

37 terremoto tettonico

38 terremoto vulcanico / o associato a fenomeni vulcanici

39 maremoto / associato al terremoto

40 leggendario o incerto (intendendo per incerto un qualunque tipo di incertezza generale: sull'avvenimento in se stesso, sulla data, o sui vari parametri ipocentrali presi nel loro insieme) 


\subsection{Bibliografia}

E' prevista anche una ricca documentazione bibliografia, intendendo come tale qualunque tipo di informazione (di cui si sia venuti a conoscenza) che abbia attinenza con il materiale del catologo (studi, ricerche, documentazioni scientifiche, comunicazioni, bollettini, cataloghi ecc...).

Per poter impostare razionalmente il criterio di segnalazione sulla scelta della fonte di informazioni, l'intera rassegna bibliografica viene suddivisa in 24 capitoli che chiameremo "tempi " con il seguente criterio: diviso l'intero periodo di tempo (dal 1450 avanti Cristo all'anno 1980) in 24 intervalli di tempo opportunamente scelti (vedi tabella 3 ), ciascun tempo (1, A, B, C,...... della bibliografia conterrà solo le documentazioni relative a quel dato intervallo di tempo.

\section{TABELLA 3}

Tabella dei 24 tempi in cui è stato suddiviso il catalogo.

\begin{tabular}{|c|c|c|c|c|c|c|}
\hline tempo & 1 & Anni & a.C. & & & Anni \\
\hline tempo & A & $1-1000$ & p.C & tempo & o & $1871-1890$ p.C. \\
\hline tempo & B & $1001-1100$ & & tempo & $P$ & $1891-1900$ \\
\hline tempo & $\mathrm{C}$ & $1101-1200$ & & tempo & $\mathrm{Q}$ & $1901-1910$ \\
\hline tempo & D & $1201-1300$ & & tempo & $\mathrm{R}$ & $1911-1920$ \\
\hline tempo & $\mathrm{E}$ & $1301-1400$ & & tempo & $\mathrm{s}$ & $1921-1930$ \\
\hline tempo & $\mathrm{F}$ & $1401-1500$ & & tempo & $\mathrm{T}$ & $1931-1940$ \\
\hline tempo & G & $1501-1600$ & & tempo & $\mathrm{U}$ & 1941-1950 \\
\hline tempo & $\mathrm{H}$ & $1601-1700$ & & tempo & V & $1951-1960$ \\
\hline tempo & I & $1701-1750$ & & tempo & $\mathrm{Z}$ & $1961-1970$ \\
\hline tempo & $\mathrm{L}$ & $1751-1800$ & & tempo & $\mathrm{X}$ & 1971-1975 \\
\hline tempo & M & $1801-1850$ & & tempo & $\mathrm{Y}$ & 1976-1980 \\
\hline tempo & $\mathrm{N}$ & $1851-1870$ & & & & \\
\hline
\end{tabular}


2. Atlante macrosismico (A/M)

Nel capitolo precedente abbiamo detto come il catalogo C.S.N. preveda anche una indicazione che faccia riferimento all'esistenza o meno delle isosiste in Atlante e/o di notizie macrosismiche.

Questa annotazione si trova ogni qualvolta sia stato possibile studiare l'aspetto macrosismico dell'evento.

Poiché anche in questo caso è di grande interesse avere una documentazione completa ed omogenea, l'ING ha intrapreso la compilazione di un Atlante Macrosismico che serva da ampliamento, integrazione e illustrazione del catalogo C.S.N. fin qui descritto.

L'Atlante riporterà, per ciascun terremoto in esso contenuto, i seguenti elementi:

2.1) il disegno delle isosiste tracciate originariamente su scala 1:500.000 fino alla isosista limite di IV-III (quando possibile);

2.2) la posizione dell'epicentro riportato dal catalogo C/A e la posizione dell'epicentro macrosismico come baricentro dell'area megasismica;

2.3) la misura (in $\mathrm{kmq}$ ) delle aree delimitate dalle is os $i$ ste chi use successive (Area di $I_{o}$ massima area di $\left(I_{o}-1\right) \ldots$, area di $I=I V$;

2.4) il valore, in $\mathrm{km}$, dei raggi dei cerchi equivalenti alle aree trovate:

$$
r_{\text {Io max }}, \quad r\left(\text { to - 1) ...; } \quad r_{\text {IV }}\right.
$$

2.5) nel caso di isosiste aperte, intendendo come tali quelle che non si possono chiudere perché l'epicentro è in mare o in prossimità delle coste (vedi figura 4), le aree misurate saranno solo quelle relative alle porzioni di territorio interessato e verranno indicate con $\mathrm{T}$; (per esempio $8630 \mathrm{~T}$ ) però in 
tal caso i raggi di propagazione saranno mis urat $i$ sui tratti delle isosiste tracciate, e non dedotti dalle aree equivalenti;

2.6) nel caso in cui l'andamento delle curve sia molto anomalo rispetto all'epicentro del sisma, sono date due serie di raggi di propagazione misurati rispettivamente lungo gli assi di massima e di minima propagazione prevalente;

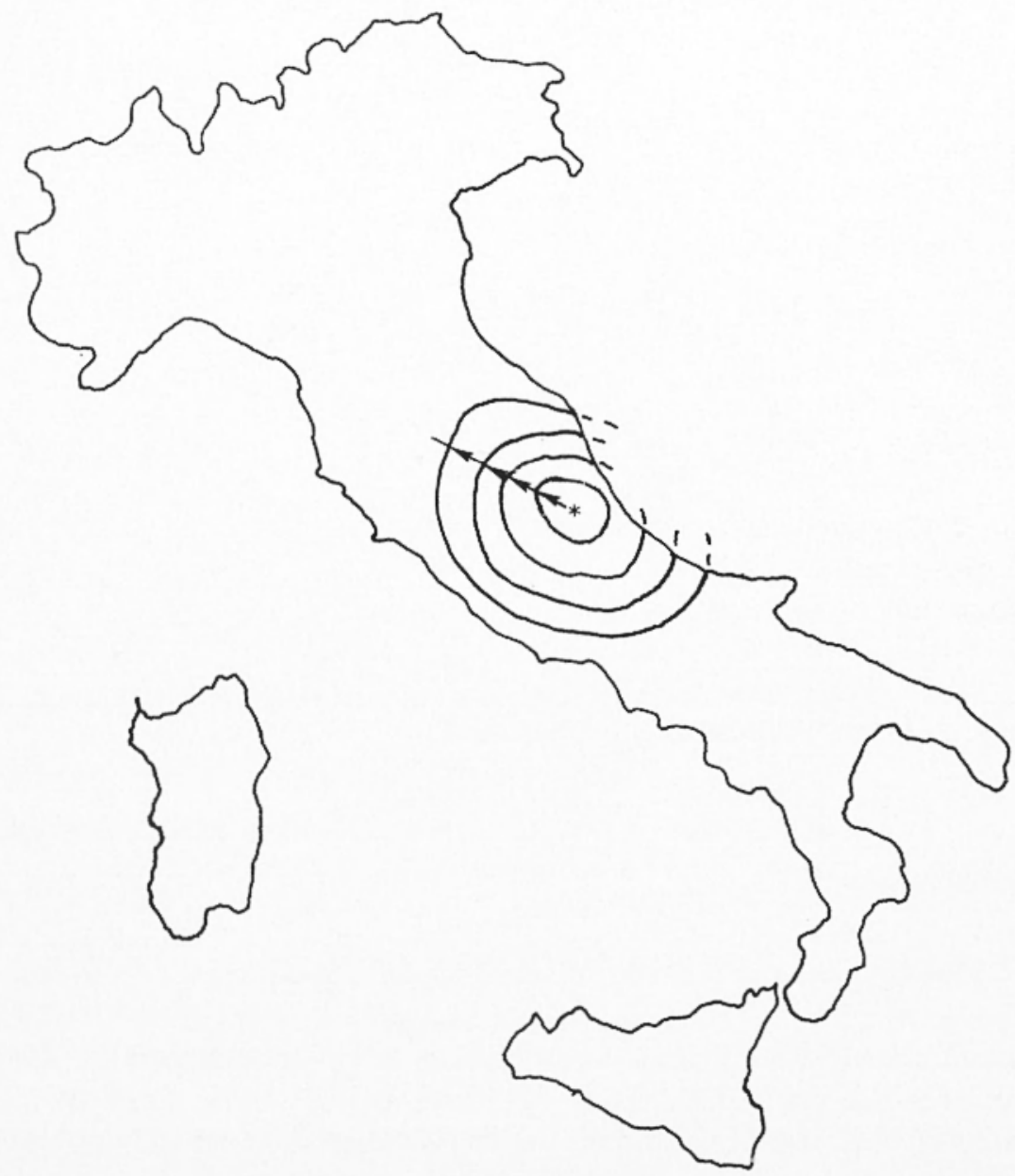

Figura 4 - Esempio di isosiste aperte. 
2.7) in quest'ultimo caso viene indicato anche l'orientamento degli assi con riferimento al Nord (meridiano condotto per l'epicentro macrosismico) ( $\alpha_{1}$ di max-propagazione; $\alpha_{2}$ di minima propagazione) (vedi figura 5 ).

N.B.: Tutti i disegni sono tracciati sopra il reticolo dei meridiani (riferiti a Greenwich) e dei paralleli, e ogni disegno riporta in piccolo una carta d'Italia su cui è indicata la posizione dell'area epicentrale.

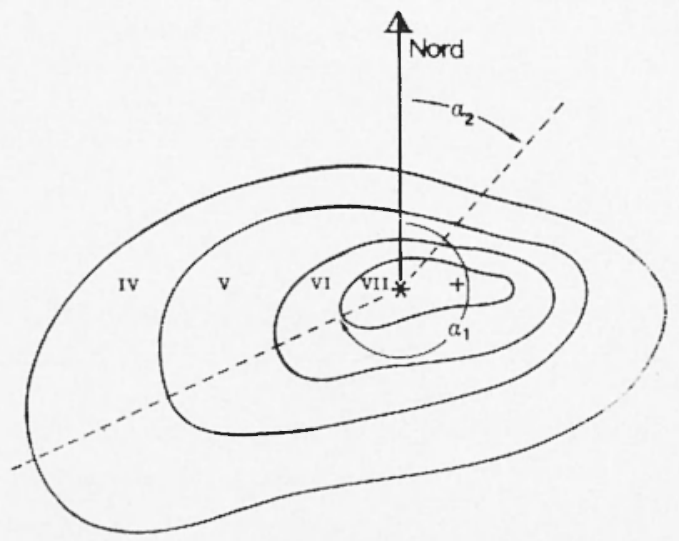

Figura 5 - Esempio di isosiste allungate e orientamento degli assi di massima e minima propagazione prevalente.

\section{OSSERVAZIONE}

Siamo consapevoli che il metodo da noi seguito per il tracciamento delle isosiste, che è quello tradizionale seguito ancora da gran parte del mondo sismologico, non trova consenzienti tutti i cultori del problema, molti dei quali si orientano verso un diverso tipo di rappresentazione; però riteniamo che un disegno che offra una visione d'insieme dell'aspetto macrosismico dell'evento, abbia ancora la sua efficacia. 







\section{Catalogo macrosismico $\mathrm{C} / \mathrm{M}$}

In questa terza parte del C.S.N. vengono riportati gli elementi di misure e di calcolo relativi all'aspetto macrosismico dei terremoti studiati nell'Atlante Macrosismico. In tal modo tutti i terremoti che nel Catalogo Analitico sono stati contrassegnati con "A" (come detto nel punto 1.7) possono venire facilmente completati anche sotto l'aspetto macrosismico con l'uso simultaneo dei due cataloghi.

In previsione di ciò, nel $\mathrm{C} / \mathrm{M}$ sono riportati: nella prima linea i seguenti parametri di riferimento:

data . . . . (anno, mese, giorno)

tempo origine Gr. (ore, minuti)

epicentro. . . . $(\varphi, \lambda)$

$h$. . . . . (in $\mathrm{km})$

intensità macros. (M.C.S.)

$M_{\mathrm{L}} M_{B} M_{\mathrm{S}}$

località geografica

elementi ripresi

dal

Catalogo Analitico

nelle linee successive vengono riportati i seguenti dati macrosismici:

a) epicentro macrosismico $(\varphi, \lambda)$ in gradi e centesimi di grado con l'attendibilità macrosismica;

b) $h$ (calcolata con metodi macrosismici) con la sua attendibilità;

c) $\left(r_{i}\right)_{c}$ (in $\left.\mathrm{km}\right)=$ raggi $r_{t}$ delle isosiste successive (secondo i criteri esposti nei punti (2.4) e (2.5) dell'Atlante macrosismico;

d) $\left(r_{1}\right)_{\max }($ in $\mathrm{km})=$ raggi $r_{t}$ delle isosiste successive misurati lungo la direzione prevalente della massima propagazione; 
e) $\left(r_{1}\right)_{\min }($ in $\mathrm{km})=$ raggi $r_{i}$ delle isosiste successive misurati lungo la direzione prevalente della minima propagazione;

f) $\left(\alpha_{1}\right)$ (in gradi) $=$ orientamento dell'asse di propagazione massima

g) $\left(\alpha_{2}\right)$ (in gradi) $=$ orientamento dell'asse di propagazione minima

\author{
f misurati dal Nord \\ $\rightarrow$ geografico, in \\ senso orario
}

Le serie di valori $d, e, f, g$ sono quelli relativi ai punti (2.6) e (2.7) dell'Atlante macrosismico.

\title{
4. SCHEDA DI LAVORO
}

Per rendere omogeneo e meno gravoso l'immane compito di catalogazione dei dati è stata studiata una "scheda di lavoro" (vedi fac-simile).

Come si vede, la scheda di lavoro prevede un largo margine di spazio in cui dovrebbe trovar posto tutto il processo di elaborazione dei dati; dai rilevamenti bibliografici alle critiche, ai calcoli, ai procedimenti che conducono infine al prodotto finito sirtetizzato nei due schemi finali della scheda analitica e della scheda macrosismica.

Questi due schemi contengono pertanto tutti gli elementi discussi nella presente nota sia per la compilazione del

\section{Catalogo analitico}

sia per la compilazione del

\section{Catalogo macrosismico}

Ovviamente, l'AtLANTE MACROSISMICo rappresenta una unità separata e indipendente, ma gli elementi suscettibili di elaborazione numerica sono trasferiti, come si è visto, nel Catalogo Macrosismico. 
Avvertenza: Dati storici e dati strumentali

\section{Dati storici}

Riteniamo utile sottolineare il fatto che lo studio presentato i stato condotto in maniera così dettagliata per poter coprire 1.utte le esigenze richieste ad una catalogazione di terremoti sufficientemente informativa.

$E^{\prime}$ ovvio tuttavia aspettarsi il meglio delle informazioni solo per quel che riguarda i tempi più recenti (grosso modo gli anni posteriori alla seconda guerra mondiale).

Per i tempi "storici " (ritenendo come tali tutti quelli che arrivano ai primi dati strumentali, ossia intorno al 1873 - data dei primi bollettini sismici pubblicati ad opera di Michele Stefano De Rossi nel "Bullettino Vulcanologico Italiano " - ci si deve accontentare della elaborazione delle notizie descrittive tratte o dalle fonti originali quando ciò è stato possibile (vale a dire sia da quelle riportate in molte delle cronache di autori raccolte dal Muratori nei suoi "Rerum Italicarum Scriptores", sia di quelle direttamente reperite di vari altri autori antichi) oppure dalle raccolte informative di studiosi di epoche più recenti.

La raccolta, l'analisi, la critica, la elaborazione delle notizie sono logicamente impostate sull'aspetto macrosismico del fenomeno: tuttavia abbiamo ritenuto utile inserire tutti i dati così elaborati in quello che abbiamo chiamato "Catalogo Analitico ", lasciando al Catalogo Macrosismico il compito di presentare, quando ciò è stato possibile, gli elementi associabili all'Atlante Macrosismico.

\section{Dati strumentali}

Dopo il 1873 cominceranno a comparire, nel Catalogo Macrosismico, oltre gli elementi descritti nel punto 3, anche gli eventuali elementi di confronto che emergono dalla duplice trattazione dell'evento, analitico-strumentale e macrosismica: vale a dire l'epicentro analitico e quello macrosismico; la profondità ipocentrale analitica e quella calcolata con metodi macrosismici, dati che molto spesso non coincidono. 


\section{BIBLIOGRAFIA}

AUtori vari: Alessio-Biagi; Bottari e altri; Caloi; Caloi-Spadea; De Panfilis-Marcelli; Feliziani-Marcelli; Iaccarino e altri; Marcelli-Pannocchia ecc.

Basili A., Marcelli L., Smriglio G., 1980. - La magnitudo macrosismica come funzione di $I_{o}$ e di h. Relazioni proposte per nove settori principali dell'area italiana. "Annali di Geofisica 》 XXXIII, (volume unico, in press.).

Calor P., 1942 - Attività sismica in Italia nel decennio 1930-1939, Firenze.

CancanI A., 1902. - Sulla distribuzione delle intensità delle repliche nei periodi sismici italiani. "Boll. Soc. Sism. Ital, ", VIII.

Casal A., Console R., De Simoni B., 1977. - Metodo di calcolo della magnitudo di terremoti registrati dai sismografi in uso agli inizi del nostro secolo. "Annali di Geofisica ", XXX, 1.

Cavasino A., 1935. - I terremoti d'Italia nel trentacinquentio 1899-1933 (Roma).

De Panfilis M., 1959. - Attività sismica in Italia dal 1953 al 1957. "Annali di Geofisica ", XII, 1.

Dominici P., Marcelli L., 1979. - Evoluzione storica della misure orarie in Italia. Suoi riflessi nello studio dei fenomeni geofisici storici, e in particolare nella catalogazione di eventi sismici. (Istituto Nazionale di Geofisica - Catalogo Sismico Nazionale - Monografia n. 13), "Annali di Geofisica », XXXII. (volume unico).

GARDNER A., KNOPOFF, 1974. - Is the Sequence of Earthquakes in Southern California, with Aftershocks, Poissonian? "Bull. Soc. Seism. Amer. » 64.

Malaroda R., RaImondi C., 1957. - Linee di dislocazione e sismicità in Italia. "Boll. di Geodesia e Scienze Affini".

Marcelli L., Pannocchis G., 1974. - Proposta per una classificazione dei dati sperimentali in sismologia: classi di attendibilità $A, B, C, D$ in funzione di $\delta \Delta$ e $\delta \mathrm{t}$. "Annali di Geofisica ", XXVII. 
Marcelli L., Pannocchia G., 1974. - Proposta per una classificazione dei dati (Esame condotto su 19 regioni dell'Area Mediterranea e sull'Italia in particolare), "Annali di Geofisica », XXXII.

Marcelli L., Peronaci M., 1979 - Analisi critica sulle profondità ipocentrali (Esame condotto su 19 regioni dell'area Mediterranea e sull'Italia in particolare). "Annali di Geofisica ", XXXII, volume unico.

Marcelli L., Spadea M.C., 1981 - Ricerca del coefficiente $\lambda=f(h)$ (della formula di Blake) valevole per il territorio italiano. Metodo rapido per calcolare "h macrosismica" con le prime tre isosiste di un terremoto. "Annali di Geofisica ", XXXIV, 1 (in press).

Mercalli G., 1883. - Geologia d'Italia parte III: Vulcani e fenomeni vulcanici, da: "I terremoti storici italiani ", cap. XII. 